\title{
Error Estimation of Perturbations Under CRI
}

\author{
Guosheng Cheng and Yuxi Fu
}

\begin{abstract}
The analysis of stability and robustness of fuzzy reasoning is an important issue in areas like intelligent systems and fuzzy control. An interesting aspect is to what extent the perturbation of input in a fuzzy reasoning scheme causes the oscillation of the output. In particular, when the error limits (restrictions) of the input values are given, what the error limits of the output values are. In this correspondence, we estimate the upper and lower bounds of the output error affected by the perturbation parameters of the input, and obtain the limits of the output values when the input values range over some interval in many fuzzy reasoning schemes under compositional rule of fuzzy inference (CRI).
\end{abstract}

Index Terms-Error estimation, fuzzy reasoning, fuzzy set, interval perturbation, simple perturbation.

\section{INTRODUCTION}

CINCE Zadeh's compositional rule of fuzzy inference [1] (CRI) was proposed, many other methods of fuzzy reasoning have been known [2]-[10]. Applications of these fuzzy reasoning techniques have been successful in various areas, especially in fuzzy control [11]. When fuzzy reasoning is applied, the stability and robustness of the fuzzy reasoning becomes one of the prominent problems. The fuzzy controllers are available to transform human expertise and subjectivity to quantitative terms, so the deviation of human expertise from its corresponding quantitative representations gives rise to the problem of the stability of fuzzy controllers [11]. The corresponding problem in fuzzy reasoning is the variance of output caused by perturbations of input. Here, the analysis of the stability of a fuzzy reasoning scheme consists of two aspects: One is how the output values of the scheme are changed by the perturbation parameters of input values. The other is how to estimate corresponding limits of output values of the scheme when oscillation limits of input values are given.

For the first question, many researchers have provided their answers [12]-[15]. Their approaches to perturbations of input are based on some proximity of fuzzy sets, using proximity measure in [12], or $\alpha$-similarity measure in [13], or maximum $\varepsilon$ perturbation in [14], or $\delta$-equality in [15]. In a sense, the problem of stability of fuzzy reasoning has been well studied. However, the effects of the perturbation parameters of the input in fuzzy reasoning schemes still call for investigation. For one thing, when a

Manuscript receivedAugust 9, 2003; revised April 13, 2005 and April 19, 2006. The work was supported by the National Distinguished Young Scientist Fund of NNSFC under Grant 60225012, by the National 973 Project under 2003CB317005, by the National Nature Science Foundation of China under Grant 60473006, by the BoShiDian Research Fund under 20010248033, by the Jiangsu Education Office Research Fund under Grant 05KJD110123, and by the Nanjing University of Information Science and Technology Research Fund under Grant QD39.

G. Cheng is with the Department of Mathematics, Nanjing University of Information Science and Technology, Nanjing 210044, China (e-mail: gshcheng@ sohu.com).

Y. Fu is with the Department of Computer Science, Shanghai Jiaotong University, Shanghai 200030, China (e-mail: fu-yx@ cs.sjtu.edu.cn).

Digital Object Identifier 10.1109/TFUZZ.2006.877333 sequence of the perturbations of the input for a fuzzy reasoning scheme has an asymptotic limit, it is obvious that the corresponding sequence of the output cannot be precisely demonstrated in the above approaches.

The correspondence is structured as follows: After introducing the concepts of the simple perturbation and the interval perturbation of the fuzzy sets, we obtain an estimation of the upper and the lower bounds of the output error affected by the simple perturbation of the input under CRI. The stability of fuzzy reasoning schemes is characterized. Also the asymptotic performance in fuzzy reasoning schemes is exhibited. Next we investigate interval perturbation of input in fuzzy reasoning and get the interval estimation of fuzzy sets inferred from CRI with some abstract implication operators and conjunction operators. Some final remarks are made in Section V.

\section{PRELIMINARIES}

The basic form of the CRI methods is as follows:

$\begin{array}{ll}\text { Antecedent : } & \text { If } x \text { is } A, \text { then } y \text { is } B \\ \text { Fact : } & x \text { is } S\end{array}$

where $A, S$ are fuzzy sets on $U$ and $B, C(A, B ; S)$ are fuzzy sets on $V$ usually defined as

$$
C(A, B ; S)(y)=\sup _{x \in U}(S(x) \subset(A(x) \rightarrow B(y)) .
$$

In the previous definition (C) is some conjunction operator, and $\rightarrow$ is an implication operator. The pair $(\mathcal{C}, \rightarrow)$ is called a scheme of fuzzy reasoning. The ordered triad $(A, B, S)$ is called an input and $C(A, B ; S)$ an output of fuzzy reasoning. Usually (C) is either min, or product, or a Lukasiewicz conjunction operator (i.e., $a \subseteq b=0 \vee(a+b-1), a, b \in[0,1])$, or a $t$-norm $\otimes$ or a $t$-conorm $\bigoplus$.

\section{A. Some Notions}

The $t$-norms $\otimes, t$-conorms $\bigoplus$, and the fuzzy complementary operations in $[0,1]$ will be fundamental for the present correspondence. A function $\otimes:[0,1] \times[0,1] \rightarrow[0,1]$ is called $t$-norm if and only if

1) $\bigotimes$ is nondecreasing in each argument;

2) $\bigotimes$ is commutative;

3) $\otimes$ is associative;

4) $\bigotimes$ has 1 as unit.

A function $\bigoplus:[0,1] \times[0,1] \rightarrow[0,1]$ is called $t$-conorm if and only if $\bigoplus$ satisfies 1 ) -3 ), as well as $4^{\prime}$ ) given as follows:

4') $\oplus$ has 0 as unit. 
A function $n:[0,1] \rightarrow[0,1]$ is called fuzzy complement if and only if

1) $n(0)=1, n(1)=0$;

2) $n$ is nonincreasing.

In this correspondence the implication operator $\rightarrow$ will stand for one of the following, where $a, b \in[0,1]$ :

- Mamdani: $a \rightarrow b=a \wedge b$;

- Kleene-Dienes: $a \rightarrow b=(1-a) \vee b$;

- Lukasiewicz: $a \rightarrow b=(1-a+b) \wedge 1$;

- Reichenbach: $a \rightarrow b=1-a+a b$;

- Zadeh: $a \rightarrow b=(1-a) \vee(a \wedge b)$;

- $I_{4}$ in [16]: $a \rightarrow b=(a \wedge b) \vee((1-a) \wedge b) \vee((1-a) \wedge$ $(1-b))$;

- $S$-implications: $a \rightarrow b=n(a) \bigoplus b$, where $n$ is a fuzzy complement, $\bigoplus$ is a $t$-conorm;

- $R$-implications: $a \rightarrow b=\sup \{\alpha \in[0,1] \mid a \otimes \alpha \leq b\}$, where $\otimes$ is a t-norm;

- $Q L$-implications: $a \rightarrow b=n(a) \bigoplus(a \bigotimes b)$, where $(\otimes, \oplus, n)$ is required to be a de Morgan triplet;

- $t$-norm implications: $a \rightarrow b=a \bigotimes b$.

Now, we introduce the concepts of simple perturbation, interval perturbation of a fuzzy set and stability of a fuzzy reasoning scheme.

Definition 1: Let $U$ be a universe of discourse, $A$ and $B$ two fuzzy sets defined on $U$. If there exists a mapping $\rho: U \rightarrow$ $[-1,1]$, such that for all $x \in U, B(x)=A(x)+\rho(x)$, then $B$ is called a simple perturbation of $A$ and $\rho$ is a factor of the perturbation of $A$.

The following definition will use the concept of fuzzy interval. If $\alpha_{1}, \alpha_{2}$ are two fuzzy sets on $U$, and for all $x \in U$, $\alpha_{1}(x) \leq \alpha_{2}(x)$, then $\left[\alpha_{1}, \alpha_{2}\right]$ is called a fuzzy interval on $U$.

Definition 2: Let $\left[\alpha_{1}, \alpha_{2}\right]$ be a fuzzy interval on $U, A$ a fuzzy set on $U$. If for all $x \in U, \alpha_{1}(x) \leq A(x) \leq \alpha_{2}(x)$, then $A$ has an interval perturbation $\left[\alpha_{1}, \alpha_{2}\right]$ on $U$, written $A \in\left[\alpha_{1}, \alpha_{2}\right]$.

Definition 3: Let $A$ and $S$ be fuzzy sets on $U, B$ a fuzzy set on $V$. Suppose $A^{\prime}, B^{\prime}$ and $S^{\prime}$ are perturbations of $A, B$ and $S$ with factor $\rho_{1}, \rho_{2}$ and $\rho_{3}$, respectively. A fuzzy reasoning scheme $($ C,$\rightarrow)$ is said to be stable if, given $\epsilon>0$, there exists $\delta>0$ such that $\left|C\left(A^{\prime}, B^{\prime} ; S^{\prime}\right)(y)-C(A, B ; S)(y)\right|<\epsilon$ on $V$ whenever $\left|\rho_{1}(x)\right|<\delta,\left|\rho_{2}(y)\right|<\delta$ and $\left|\rho_{3}(x)\right|<\delta$.

A perturbation sequence $\left\{\left(A_{n}^{\prime}, B_{n}^{\prime}, S_{n}^{\prime}\right)\right\}$ of an input $(A, B, S)$ with a corresponding sequence $\left\{\rho_{1 n}(x), \rho_{2 n}(y), \rho_{3 n}(x)\right\}$ of factors in some fuzzy reasoning scheme is said to be (asymptotic) stable if, given $\epsilon>0$, there exist some natural number $n_{0}$, and $\delta>0$ such that $\left|C\left(A_{n}^{\prime}, B_{n}^{\prime} ; S_{n}^{\prime}\right)(y)-C(A, B ; S)(y)\right|<\epsilon$ on $V$ for all $n>n_{0}$, $\left|\rho_{1 n}(x)\right|<\delta,\left|\rho_{2 n}(y)\right|<\delta$, and $\left|\rho_{3 n}(x)\right|<\delta$.

Definition 4: Let $A \in\left[\alpha_{1}, \alpha_{2}\right], S \in\left[\gamma_{1}, \gamma_{2}\right]$ be fuzzy sets on $U, B \in\left[\beta_{1}, \beta_{2}\right]$ a fuzzy set on $V$. A fuzzy reasoning scheme (C), $\rightarrow$ ) is said to be stable if, given $\epsilon>0$, there exist a fuzzy interval $\left[\lambda_{1}, \lambda_{2}\right]$ on $V$ and $\delta>0$, such that for each $y \in V$, $\lambda_{2}(y)-\lambda_{1}(y)<\epsilon$, and $C(A, B ; S) \in\left[\lambda_{1}, \lambda_{2}\right]$ whenever $\alpha_{2}(x)-\alpha_{1}(x)<\delta, \beta_{2}(y)-\beta_{1}(y)<\delta$, and $\gamma_{2}(x)-\gamma_{1}(x)<\delta$.

\section{B. Related Work}

1) Pappis' Work: Pappis introduced the approximately equal of two fuzzy sets on $U$ in [12], i.e., let $A_{1}$ and $A_{2}$ be fuzzy sets on $U$, if then $A_{1}$ and $A_{2}$ are said to be approximately equal, denoted by $A_{1} \approx A_{2}$. $\varepsilon$ is said to be a proximity measure of $A_{1}$ and $A_{2}$. The Pappis' result is as follows.

Let $A, A_{1}$ and $A_{2}$ be fuzzy sets on $U$, and $R, R_{1}$ and $R_{2}$ fuzzy relations from $U$ to $V$. Then

- $A_{1} \approx A_{2}$ implies $A_{1} \circ R \approx A_{2} \circ R$;

- $R_{1} \approx R_{2}$ implies $A \circ R_{1} \approx A \circ R_{2}$;

where $\circ$ is the max-min composition.

We see that this result actually addresses the stability of some fuzzy reasoning schemes when $A, B$, and $S$ of the input $(A, B ; S)$ are perturbed, respectively.

2) Hong and Hwang's Work: Hong and Hwang defined the $\alpha$-similarity of two fuzzy sets on $U$ in [13], i.e., let $A_{1}$ and $A_{2}$ be fuzzy sets on $U$, if

$$
1-\sup _{x \in U}\left|A_{1}(x)-A_{2}(x)\right| \geq \alpha, \quad \alpha \in[0,1]
$$

then $A_{1}$ and $A_{2}$ are said to be $\alpha$-similar, denoted by $A_{1} \approx_{\alpha} A_{2}$.

Hong and Hwang generalized the Pappis' result to be the following.

Let $A_{1}$ and $A_{2}$ be fuzzy sets on $U$, and $R_{1}$ and $R_{2}$ fuzzy relations from $U$ to $V$. If $A_{1} \approx_{\alpha} A_{2}$ and $R_{1} \approx_{\alpha} R_{2}$, then $A_{1} \circ R_{1} \approx_{\alpha} A_{2} \circ R_{2}$.

By means of this result, the stability of some fuzzy reasoning schemes is obtained when $A, B$, and $S$ of the input $(A, B ; S)$ are all perturbed.

3) Ying's Work: Ying introduced the concept of maximum perturbation of fuzzy set in [14] as follows.

Let $A_{1}$ and $A_{2}$ be fuzzy sets on $U$ and $\epsilon \in[0,1]$. If for each $x \in U,\left|A_{1}(x)-A_{2}(x)\right| \leq \epsilon$, then $A_{2}$ is called a maximum $\epsilon$ perturbation of $A_{1}$.

One of the main results in [14] was to obtain the maximum perturbation $C\left(A_{2}, B_{2} ; S_{2}\right)$ of the output $C\left(A_{1}, B_{1} ; S_{1}\right)$ when all of $A_{1}, B_{1}$ and $S_{1}$ of the input $\left(A_{1}, B_{1}, S_{1}\right)$ have the $\epsilon$ maximum perturbations. See [14] for more details.

Evidently, the stability of some fuzzy reasoning schemes may be precisely characterized by the Ying's result.

4) Cai's Work: Cai used the term " $\delta$-equality" in [15] as follows.

Let $A_{1}$ and $A_{2}$ be fuzzy sets on $U$. Then, $A_{1}$ and $A_{2}$ are said to be $\delta$-equal, if $\sup _{x \in U}\left|A_{1}(x)-A_{2}(x)\right| \leq 1-\delta, \delta \in[0,1]$.

Cai investigated $\delta$-equalities for some implication operators, $t$-conorm, fuzzy relations and generalized modus pollens in [15].

The stability and instability of some fuzzy reasoning schemes are easily addressed by the Cai's results.

\section{Two Lemmas}

Lemma 1 can be easily established.

Lemma 1: Let $f$ and $g$ be bounded, real-valued functions defined on $U$ (or $V$ ), $A$ and $B$ fuzzy sets on $U$. Then, the following properties hold:

1) $f \vee g=\max \{f, g\}=(f+g) / 2+|f-g| / 2$;

2) $f \wedge g=\min \{f, g\}=(f+g) / 2-|f-g| / 2$;

3) $-|f-g| \leq|f|-|g| \leq|f-g|$;

4) $\inf _{x \in U} A(x)+\inf _{x \in U} B(x) \leq \inf _{x \in U}(A(x)+B(x))$;

5) $\sup _{x \in U}(A(x)+B(x)) \leq \sup _{x \in U} A(x)+\sup _{x \in U} B(x)$;

6) $\sup _{x \in U} A(x)=-\inf _{x \in U}(-A(x))$;

7) $\inf _{x \in U}(A(x) \wedge B(x))=\inf _{x \in U} A(x) \wedge \inf _{x \in U} B(x)$, $\inf _{x \in U}(A(x) \vee B(x)) \geq \inf _{x \in U} A(x) \vee \inf _{x \in U} B(x)$; 
8) $\sup _{x \in U}(A(x) \wedge B(x)) \leq \sup _{x \in U} A(x) \wedge \sup _{x \in U} B(x)$, $\sup _{x \in U}(A(x) \vee B(x))=\sup _{x \in U} A(x) \vee \sup _{x \in U} B(x)$.

If some implication operators are perturbed, one gets the following.

Lemma 2: Let $A$ be a fuzzy set on $U, B$ a fuzzy set on $V$. $\rho_{1}$, and $\rho_{2}$ are factors of perturbation of $A$ and $B$, respectively. Let $\Delta I(x, y)$ stand for $\left(\left(A(x)+\rho_{1}(x)\right) \rightarrow\left(B(y)+\rho_{2}(y)\right)\right)-$ $(A(x) \rightarrow B(y))$. Then, the following inequalities hold.

1) If $\rightarrow$ is Mamdani implication, then

$$
\rho_{1}(x) \wedge \rho_{2}(y) \leq \Delta I(x, y) \leq \rho_{1}(x) \vee \rho_{2}(y) .
$$

2) If $\rightarrow$ is Kleene-Dienes implication, then

$$
\left(-\rho_{1}(x)\right) \wedge \rho_{2}(y) \leq \Delta I(x, y) \leq\left(-\rho_{1}(x)\right) \vee \rho_{2}(y) .
$$

3) If $\rightarrow$ is Lukasiewicz implication, then

$$
\begin{aligned}
(-1) & \vee\left(0 \wedge\left(\rho_{2}(y)-\rho_{1}(x)\right)\right) \leq \Delta I(x, y) \\
\leq 1 & \wedge\left(0 \vee\left(\rho_{2}(y)-\rho_{1}(x)\right)\right) .
\end{aligned}
$$

4) If $\rightarrow$ is Reichenbach implication, then

$$
\begin{aligned}
(-1) & \vee\left(0 \wedge \rho_{2}(y)-0 \vee \rho_{1}(x)\right) \leq \Delta I(x, y) \\
\leq 1 & \wedge\left(0 \vee \rho_{2}(y)-0 \wedge \rho_{1}(x)\right) .
\end{aligned}
$$

5) If $\rightarrow$ is Zadeh implication, then

$$
\begin{aligned}
\left(-\rho_{1}(x)\right) & \wedge \rho_{1}(x) \wedge \rho_{2}(y) \leq \Delta I(x, y) \\
& \leq\left(-\rho_{1}(x)\right) \vee \rho_{1}(x) \vee \rho_{2}(y) .
\end{aligned}
$$

6) If $\rightarrow$ is $I_{4}$ in [16], then

$$
\begin{aligned}
\rho_{1}(x) & \wedge \rho_{2}(y) \wedge\left(-\rho_{1}(x) \vee \rho_{2}(y)\right) \\
& \leq \Delta I(x, y) \leq \rho_{1}(x) \\
& \vee \rho_{2}(y) \vee\left(-\rho_{1}(x) \wedge \rho_{2}(y)\right) .
\end{aligned}
$$

Proof: We only prove 5). The proofs of the other cases are similar. For $x \in U, y \in V$, one has, by 2) of Lemma 1, that

$$
\begin{aligned}
&\left(A(x)+\rho_{1}(x)\right) \wedge\left(B(y)+\rho_{2}(y)\right) \\
&= \frac{A(x)+\rho_{1}(x)+B(y)+\rho_{2}(y)}{2} \\
&-\frac{\left|A(x)+\rho_{1}(x)-\left(B(y)+\rho_{2}(y)\right)\right|}{2} \\
&= \frac{\rho_{1}(x)+\rho_{2}(y)}{2} \\
&-\left(\frac{\left|A(x)+\rho_{1}(x)-\left(B(y)+\rho_{2}(y)\right)\right|}{2}\right.
\end{aligned}
$$

$$
\begin{gathered}
\left.-\frac{|A(x)-B(y)|}{2}\right) \\
+\frac{A(x)+B(y)}{2}-\frac{|A(x)-B(y)|}{2} \\
=\frac{\rho_{1}(x)+\rho_{2}(y)}{2} \\
-\left(\frac{\left|A(x)+\rho_{1}(x)-\left(B(y)+\rho_{2}(y)\right)\right|}{2}\right. \\
\left.-\frac{|A(x)-B(y)|}{2}\right) \\
+A(x) \wedge B(y) .
\end{gathered}
$$

Again by 3) of Lemma 1, one has that

$$
\begin{aligned}
\left(A(x)+\rho_{1}(x)\right) \wedge & \left(B(y)+\rho_{2}(y)\right) \\
\leq & \frac{\rho_{1}(x)+\rho_{2}(y)}{2} \\
& +\frac{\left|\rho_{1}(x)-\rho_{2}(y)\right|}{2}+A(x) \wedge B(y) \\
= & \rho_{1}(x) \vee \rho_{2}(y)+A(x) \wedge B(y)
\end{aligned}
$$

and that

$$
\begin{aligned}
\left(A(x)+\rho_{1}(x)\right) \wedge & \left(B(y)+\rho_{2}(y)\right) \\
\geq & \frac{\rho_{1}(x)+\rho_{2}(y)}{2}-\frac{\left|\rho_{1}(x)-\rho_{2}(y)\right|}{2} \\
& +A(x) \wedge B(y) \\
= & \rho_{1}(x) \wedge \rho_{2}(y)+A(x) \wedge B(y) .
\end{aligned}
$$

Therefore by 1) -3 ) of Lemma 1, one has the equation shown at the bottom of the page, and that

$$
\begin{aligned}
\left(1-A(x)-\rho_{1}(x)\right) \vee & \left(\left(A(x)+\rho_{1}(x)\right) \wedge\left(B(y)+\rho_{2}(y)\right)\right) \\
\geq & \left(1-A(x)-\rho_{1}(x)\right) \\
\vee & \left(\rho_{1}(x) \wedge \rho_{2}(y)+A(x) \wedge B(y)\right) \\
\geq & \left(-\rho_{1}(x)\right) \wedge \rho_{1}(x) \wedge \rho_{2}(y) \\
& \quad+(1-A(x)) \vee(A(x) \wedge B(y)) .
\end{aligned}
$$

It follows that

$$
\begin{aligned}
\left(-\rho_{1}(x)\right) \wedge \rho_{1}(x) \wedge \rho_{2}(y) & \leq \Delta I(x, y) \\
& \leq\left(-\rho_{1}(x)\right) \vee \rho_{1}(x) \vee \rho_{2}(y) .
\end{aligned}
$$

\section{Simple Perturbation}

In this section, when the input values are simply perturbed, we estimate the upper and lower bounds of the output values in

$$
\begin{aligned}
\left(1-A(x)-\rho_{1}(x)\right) \vee & \left(\left(A(x)+\rho_{1}(x)\right) \wedge\left(B(y)+\rho_{2}(y)\right)\right) \\
\leq & \left(1-A(x)-\rho_{1}(x)\right) \\
\vee & \left(\rho_{1}(x) \vee \rho_{2}(y)+A(x) \wedge B(y)\right) \\
= & \frac{1-A(x)-\rho_{1}(x)+\rho_{1}(x) \vee \rho_{2}(y)+A(x) \wedge B(y)}{2} \\
& +\frac{\left|1-A(x)-\rho_{1}(x)-\left(\rho_{1}(x) \vee \rho_{2}(y)+A(x) \wedge B(y)\right)\right|}{2} \\
\leq & \left(-\rho_{1}(x)\right) \vee \rho_{1}(x) \vee \rho_{2}(y)+(1-A(x)) \vee(A(x) \wedge B(y))
\end{aligned}
$$


fuzzy reasoning according to some choice of conjunction and implication operators.

Let $\triangle(y)$ be $C\left(A^{\prime}, B^{\prime} ; S^{\prime}\right)(y)-C(A, B ; S)(y)$, where $A^{\prime}(x)=A(x)+\rho_{1}(x)$ and $S^{\prime}(x)=S(x)+\rho_{3}(x)$ are the perturbations of fuzzy sets $A$ and $S$ on $U$, and $B^{\prime}(y)=B(y)+\rho_{2}(y)$ is the perturbation of the fuzzy set $B$ on $V$. For simplicity, we denote $\sup _{x \in U} \rho_{1}(x), \inf _{x \in U} \rho_{1}(x), \sup _{x \in U} \rho_{3}(x)$, and $\inf _{x \in U} \rho_{3}(x)$ by $s \rho_{1}, i \rho_{1}, s \rho_{3}$ and $i \rho_{3}$, respectively.

The next three theorems describe the main results.

Theorem 1: Let $A$ and $S$ be fuzzy sets on $U$, and $B$ a fuzzy set on $V$. Suppose $A^{\prime}, B^{\prime}$ and $S^{\prime}$ are perturbations of $A, B$ and $S$ with factors $\rho_{1}, \rho_{2}$ and $\rho_{3}$, respectively. If (C) is min then the following properties hold.

1) If $\rightarrow$ is Mamdani implication, then

$$
i \rho_{1} \wedge \rho_{2}(y) \wedge i \rho_{3} \leq \triangle(y) \leq s \rho_{1} \vee \rho_{2}(y) \vee s \rho_{3} .
$$

2) If $\rightarrow$ is Kleene-Dienes implication, then

$$
\left(-s \rho_{1}\right) \wedge \rho_{2}(y) \wedge i \rho_{3} \leq \triangle(y) \leq\left(-i \rho_{1}\right) \vee \rho_{2}(y) \vee s \rho_{3} .
$$

3) If $\rightarrow$ is Lukasiewiz implication, then

$$
\begin{aligned}
(-1) & \vee\left(0 \wedge\left(\rho_{2}(y)-s \rho_{1}\right) \wedge i \rho_{3}\right) \\
& \leq \triangle(y) \leq 1 \wedge\left(0 \vee\left(\rho_{2}(y)-i \rho_{1}\right) \vee s \rho_{3}\right) .
\end{aligned}
$$

4) If $\rightarrow$ is Reichenbach implication, then

$$
\begin{aligned}
(-1) & \vee\left(i \rho_{3} \wedge\left(0 \wedge \rho_{2}(y)-0 \vee s \rho_{1}\right)\right) \\
\leq \triangle(y) & \leq 1 \wedge\left(s \rho_{3} \vee\left(0 \vee \rho_{2}(y)-0 \wedge i \rho_{1}\right)\right) .
\end{aligned}
$$

5) If $\rightarrow$ is Zadeh implication, then

$$
\begin{aligned}
\left(-s \rho_{1}\right) & \wedge i \rho_{1} \wedge \rho_{2}(y) \wedge i \rho_{3} \\
& \leq \triangle(y) \leq\left(-i \rho_{1}\right) \vee s \rho_{1} \vee \rho_{2}(y) \vee s \rho_{3} .
\end{aligned}
$$

6) If $\rightarrow$ is $I_{4}$ in [16], then

$$
\begin{aligned}
i \rho_{1} & \wedge \rho_{2}(y) \\
\leq & \wedge i \rho_{3} \wedge\left(-s \rho_{1} \vee \rho_{2}(y)\right) \\
\leq & \leq s \rho_{1} \vee \rho_{2}(y) \vee s \rho_{3} \vee\left(-i \rho_{1} \wedge \rho_{2}(y)\right) .
\end{aligned}
$$

7) If $\rightarrow$ is Zadeh implication, then

$$
\begin{aligned}
\left(-s \rho_{1}\right) & \wedge i \rho_{1} \wedge \rho_{2}(y) \wedge i \rho_{3} \\
& \leq \triangle(y) \leq\left(-i \rho_{1}\right) \vee s \rho_{1} \vee \rho_{2}(y) \vee s \rho_{3} .
\end{aligned}
$$

Proof: We only provide the proof of 3). The proofs of the other cases are similar. By 4)-6) of Lemma 1, one obtains that

$$
\begin{aligned}
\triangle(y) \geq \inf _{x \in U}( & \left(S(x)+\rho_{3}(x)\right) \\
& \wedge\left(\left(A(x)+\rho_{1}(x)\right) \rightarrow\left(B(y)+\rho_{2}(y)\right)\right) \\
& -S(x) \wedge(A(x) \rightarrow B(y)))
\end{aligned}
$$

and

$$
\begin{aligned}
\triangle(y) \leq \sup _{x \in U}( & \left(S(x)+\rho_{3}(x)\right) \\
& \wedge\left(\left(A(x)+\rho_{1}(x)\right) \rightarrow\left(B(y)+\rho_{2}(y)\right)\right) \\
& -S(x) \wedge(A(x) \rightarrow B(y))) .
\end{aligned}
$$

By 3) of Lemma 2, one has

$$
\begin{aligned}
\left(S(x)+\rho_{3}(x)\right) \wedge & \left(\left(A(x)+\rho_{1}(x)\right) \rightarrow\left(B(y)+\rho_{2}(y)\right)\right) \\
& -S(x) \wedge(A(x) \rightarrow B(y)) \\
\leq & \left(S(x)+\rho_{3}(x)\right) \\
& \left(0 \vee\left(\rho_{2}(y)-\rho_{1}(x)\right)+A(x) \rightarrow B(y)\right) \\
& -S(x) \wedge(A(x) \rightarrow B(y)) \\
\leq & 0 \vee\left(\rho_{2}(y)-\rho_{1}(x)\right) \vee \rho_{3}(x)
\end{aligned}
$$

and

$$
\begin{aligned}
\left(S(x)+\rho_{3}(x)\right) \wedge & \left(\left(A(x)+\rho_{1}(x)\right) \rightarrow\left(B(y)+\rho_{2}(y)\right)\right) \\
& -S(x) \wedge(A(x) \rightarrow B(y)) \\
\geq & \left(S(x)+\rho_{3}(x)\right) \wedge\left(0 \wedge\left(\rho_{2}(y)-\rho_{1}(x)\right)\right. \\
& +A(x) \rightarrow B(y)) \\
& -S(x) \wedge(A(x) \rightarrow B(y)) \\
\geq & 0 \wedge\left(\rho_{2}(y)-\rho_{1}(x)\right) \wedge \rho_{3}(x) .
\end{aligned}
$$

Obviously, $-1 \leq \triangle(y) \leq 1$. We are done by applying (7) and (8) of Lemma.

When (c) is product, we have the following results.

Theorem 2: Let (c) be product. The other conditions are the same as in Theorem 1. Then, the following inequalities hold.

1) If $\rightarrow$ is Mamdani implication, then

$$
\begin{aligned}
(-1) & \vee\left(0 \wedge i \rho_{1} \wedge \rho_{2}(y)+0 \wedge i \rho_{3}\right) \\
\leq & \triangle(y) \leq 1 \wedge\left(0 \vee s \rho_{1} \vee \rho_{2}(y)+0 \vee s \rho_{3}\right) .
\end{aligned}
$$

2) If $\rightarrow$ is Kleene-Dienes implication, then

$$
\begin{aligned}
(-1) & \vee\left(0 \wedge\left(-s \rho_{1}\right) \wedge \rho_{2}(y)+0 \wedge i \rho_{3}\right) \\
\leq & \triangle(y) \leq 1 \wedge\left(0 \vee\left(-i \rho_{1}\right) \vee \rho_{2}(y)+0 \vee s \rho_{3}\right) .
\end{aligned}
$$

3) If $\rightarrow$ is Lukasiewiz implication, then

$$
\begin{aligned}
(-1) & \vee\left(0 \wedge\left(\rho_{2}(y)-s \rho_{1}\right)+0 \wedge i \rho_{3}\right) \\
\leq \triangle & \triangle(y) \leq 1 \wedge\left(0 \vee\left(\rho_{2}(y)-i \rho_{1}\right)+0 \wedge s \rho_{3}\right) .
\end{aligned}
$$

4) If $\rightarrow$ is Reichenbach implication, then

$$
\begin{aligned}
(-1) & \vee\left(0 \wedge\left(-s \rho_{1}\right)+0 \wedge \rho_{2}(y)+0 \wedge \rho_{3}\right) \\
\leq & \triangle(y) \leq 1 \wedge\left(0 \vee\left(-i \rho_{1}\right)+0 \vee \rho_{2}(y)+0 \vee s \rho_{3}\right) .
\end{aligned}
$$

5) If $\rightarrow$ is Zadeh implication, then

$$
\begin{aligned}
(-1) & \vee\left(0 \wedge\left(-s \rho_{1}\right) \wedge i \rho_{1} \wedge \rho_{2}(y)+0 \wedge i \rho_{3}\right) \\
\leq \triangle(y) \leq 1 & \wedge\left(0 \vee\left(-i \rho_{1}\right) \vee s \rho_{1} \vee \rho_{2}(y)+0 \vee s \rho_{3}\right) .
\end{aligned}
$$

6) If $\rightarrow$ is $I_{4}$ as defined in [16], then

$$
\begin{aligned}
(-1) & \vee\left(0 \wedge i \rho_{1} \wedge \rho_{2}(y) \wedge\left(-s \rho_{1} \vee \rho_{2}(y)\right)+0 \wedge i \rho_{3}\right) \\
& \leq \triangle(y) \leq 1 \\
& \wedge\left(0 \vee s \rho_{1} \vee \rho_{2}(y) \vee\left(-i \rho_{1} \wedge \rho_{2}(y)\right)+0 \vee s \rho_{3}\right) .
\end{aligned}
$$

Proof: We only give the proof of 2). To start with, one has by 2) of Lemma 2 that

$$
\begin{aligned}
& \left(S(x)+\rho_{3}(x)\right)\left(\left(A(x)+\rho_{1}(x)\right) \rightarrow\left(B(y)+\rho_{2}(y)\right)\right) \\
& \quad=\left(S(x)+\rho_{3}(x)\right)\left(\left(1-A(x)-\rho_{1}(x)\right) \vee\left(B(y)+\rho_{2}(y)\right)\right)
\end{aligned}
$$




$$
\begin{aligned}
& \leq\left(S(x)+\rho_{3}(x)\right)\left(\left(-\rho_{1}(x)\right) \vee \rho_{2}(y)+A(x) \rightarrow B(y)\right) \\
& =\left(S(x)+\rho_{3}(x)\right)\left(-\rho_{1}(x)\right) \\
& \vee \rho_{2}(y)+(A(x) \rightarrow B(y)) \rho_{3}(x)+S(x)(A(x) \rightarrow B(y)) .
\end{aligned}
$$

Then

$$
\triangle(y) \leq 0 \vee\left(-i \rho_{1}\right) \vee \rho_{2}(y)+0 \vee s \rho_{3}
$$

and $\triangle(y) \leq 1$. Therefore

$$
\triangle(y) \leq 1 \wedge\left(0 \vee\left(-i \rho_{1}\right) \vee \rho_{2}(y)+0 \vee s \rho_{3}\right)
$$

The rest of the proof of is similar.

If (c) is the Lukasiewicz conjunction, then we have the following result.

Theorem 3: Let (c) be Lukasiewicz conjunction. The other conditions are the same as in Theorem 1. Then, the following properties hold.

1) If $\rightarrow$ is Mamdani implication, then

$$
\begin{aligned}
(-1) & \vee\left(0 \wedge\left(i \rho_{1} \wedge \rho_{2}(y)+i \rho_{3}\right)\right) \\
& \leq \triangle(y) \leq 1 \wedge\left(0 \vee\left(s \rho_{1} \vee \rho_{2}(y)+s \rho_{3}\right)\right) .
\end{aligned}
$$

2) If $\rightarrow$ is Kleene-Dienes implication, then

$$
\begin{aligned}
&(-1) \vee\left(0 \wedge\left(\left(-s \rho_{1}\right) \wedge \rho_{2}(y)+i \rho_{3}\right)\right) \\
& \leq \triangle(y) \leq 1 \wedge\left(0 \vee\left(\left(-i \rho_{1}\right) \vee \rho_{2}(y)+s \rho_{3}\right)\right) .
\end{aligned}
$$

3) If $\rightarrow$ is Lukasiewiz implication, then

$$
\begin{aligned}
(-1) & \vee\left(0 \wedge\left(0 \wedge\left(\rho_{2}(y)-s \rho_{1}\right)+i \rho_{3}\right)\right) \\
\leq \triangle & \triangle(y) \leq 1 \wedge\left(0 \vee\left(0 \vee\left(\left(\rho_{2}(y)-i \rho_{1}\right)+s \rho_{3}\right)\right)\right) .
\end{aligned}
$$

4) If $\rightarrow$ is Reichenbach implication, then

$$
\begin{aligned}
(-1) & \vee\left(-0 \vee s \rho_{1}+0 \wedge \rho_{2}(y)+0 \wedge i \rho_{3}\right) \\
& \leq \triangle(y) \leq 1 \wedge\left(-0 \wedge i \rho_{1}+0 \vee \rho_{2}(y)+0 \vee s \rho_{3}\right) .
\end{aligned}
$$

5) If $\rightarrow$ is Zadeh implication, then

$$
\begin{aligned}
(-1) & \vee\left(0 \wedge\left(i \rho_{1} \wedge \rho_{2}(y) \wedge\left(-s \rho_{1}\right)+i \rho_{3}\right)\right) \\
\leq & \triangle(y) \leq 1 \wedge\left(0 \vee\left(s \rho_{1} \vee \rho_{2}(y) \vee\left(-i \rho_{1}\right)+s \rho_{3}\right)\right) .
\end{aligned}
$$

6) If $\rightarrow$ is $I_{4}$ as defined in [16], then

$$
\begin{aligned}
(-1) & \vee\left(0 \wedge\left(i \rho_{1} \wedge \rho_{2}(y) \wedge\left(-s \rho_{1} \vee \rho_{2}(y)\right)+i \rho_{3}\right)\right) \\
& \leq \triangle(y) \leq 1 \\
& \wedge\left(0 \vee\left(s \rho_{1} \vee \rho_{2}(y) \vee\left(-i \rho_{1} \wedge \rho_{2}(y)\right)+s \rho_{3}\right)\right) .
\end{aligned}
$$

Proof: Now, we only verify that 4) holds. Since the equation shown at the bottom of the page holds, one has

$$
\begin{aligned}
\triangle(y) \leq & \sup _{x \in U}\left\{0 \vee \rho_{3}(x)\right. \\
& \left.-(1-B(y)) \rho_{1}(x)+\left(A(x)+\rho_{1}(x)\right) \rho_{2}(y)\right\} \\
\leq & -0 \wedge i \rho_{1}+0 \vee \rho_{2}(y)+0 \vee s \rho_{3} .
\end{aligned}
$$

It is clear that $\triangle(y) \leq 1$. Thus

$$
\triangle(y) \leq 1 \wedge\left(-0 \wedge i \rho_{1}+0 \vee \rho_{2}(y)+0 \vee s \rho_{3}\right)
$$

We omit the details of the rest of the proof of 4) because of similarity.

The proofs of the other assertions are similar.

Let $A, S$ be fuzzy sets on $U, B$ a fuzzy set on $V$. Suppose that there exists a perturbation sequence $\left\{\left(A_{n}^{\prime}, B_{n}^{\prime}, S_{n}^{\prime}\right)\right\}$ of an input $(A, B, S)$ with a corresponding sequence $\left\{\rho_{1 n}(x), \rho_{2 n}(y), \rho_{3 n}(x)\right\}$ of factors in some fuzzy reasoning scheme. If $\rho_{1 n}(x), \rho_{2 n}(y), \rho_{3 n}(x)$ satisfy

$$
\begin{aligned}
& \lim _{n \rightarrow \infty} \sup _{x \in U}\left|\rho_{k n}(x)\right|=0, \text { where } k=1 \text { or } 3 \\
& \lim _{n \rightarrow \infty} \sup _{y \in V}\left|\rho_{2 n}(y)\right|=0 .
\end{aligned}
$$

Then, by previous theorems, we have

$$
\lim _{n \rightarrow \infty} C\left(A_{n}^{\prime}, B_{n}^{\prime} ; S_{n}^{\prime}\right)(y)=C(A, B ; S)(y) .
$$

On the other hand, if there are a small positive real number $\epsilon$, and mappings $\theta_{k}(x): U \rightarrow[-1,1]$ for $k=1,3$ or $\theta_{2}(y)$ : $V \rightarrow[-1,1]$ such that $\rho_{k}(x)=\epsilon \theta_{k}(x)$, where $k=1,3$, and $\rho_{2}(y)=\epsilon \theta_{2}(y)$, then

$$
\lim _{\epsilon \rightarrow 0} C\left(A^{\prime}, B^{\prime} ; S^{\prime}\right)(y)=C(A, B ; S)(y) .
$$

$$
\begin{aligned}
(S(x) & \left.+\rho_{3}(x)\right) \mathcal{C}\left(\left(A(x)+\rho_{1}(x)\right) \rightarrow\left(B(y)+\rho_{2}(y)\right)\right) \\
= & 0 \vee\left(S(x)+\rho_{3}(x)+1-\left(A(x)+\rho_{1}(x)\right)+\left(A(x)+\rho_{1}(x)\right)\left(B(y)+\rho_{2}(y)\right)-1\right) \\
= & \left(\frac{S(x)+\rho_{3}(x)+1-A(x)-\rho_{1}(x)}{2}+\frac{\left(A(x)+\rho_{1}(x)\right)\left(B(y)+\rho_{2}(y)\right)-1}{2}\right) \\
& +\frac{\left|S(x)+\rho_{3}(x)+1-A(x)-\rho_{1}(x)+\left(A(x)+\rho_{1}(x)\right)\left(B(y)+\rho_{2}(y)\right)-1\right|}{2} \\
\leq & 0 \vee\left(\rho_{3}(x)-(1-B(y)) \rho_{1}(x)+\left(A(x)+\rho_{1}(x)\right) \rho_{2}(y)\right) \\
\quad & +S(x) \subseteq(A(x) \rightarrow B(y))
\end{aligned}
$$


Now it is clear that the aformentioned fuzzy reasoning schemes using Zadeh's CRI methods are stable. Their asymptotic performance has been demonstrated.

\section{INTERVAL PERTURBATION}

In this section, $C(A, B ; S)(y)=\sup _{x \in U}(S(x)(\mathcal{C}(A(x) \rightarrow$ $B(y))$, where (C) is some $t$-norm or $t$-conorm, and $\rightarrow$ is one of $S$-implications, $R$-implications, $Q L$-implications, or $t$-norm implications. We give the estimations of the limits of the output with the interval perturbation of the input in CRI methods.

Let $\left[\alpha_{1}, \alpha_{2}\right],\left[\gamma_{1}, \gamma_{2}\right]$ be fuzzy intervals on $U,\left[\beta_{1}, \beta_{2}\right]$ a fuzzy interval on $V, s \alpha_{2}, i \alpha_{1}, s \gamma_{2}$, and $i \gamma_{1}$ stand for $\sup _{x \in U} \alpha_{2}(x)$, $\inf _{x \in U} \alpha_{1}(x), \sup _{x \in U} \gamma_{2}(x), \inf _{x \in U} \gamma_{1}(x)$, respectively. The main results are as follows.

Theorem 4: Suppose that $A \in\left[\alpha_{1}, \alpha_{2}\right], S \in\left[\gamma_{1}, \gamma_{2}\right]$ are fuzzy sets on $U, B \in\left[\beta_{1}, \beta_{2}\right]$ is a fuzzy set on $V$, and (C) is either a $t$-norm or a $t$-conorm.

1) If $\rightarrow$ is one of $S$-implications, $R$-implications, then

$$
\begin{aligned}
i \gamma_{1} @\left(s \alpha_{2} \rightarrow \beta_{1}(y)\right) & \leq C(A, B ; S)(y) \\
& \leq s \gamma_{2}\left(\mathcal{C}\left(i \alpha_{1} \rightarrow \beta_{2}(y)\right) .\right.
\end{aligned}
$$

2) If $\rightarrow$ is an $Q L$-implication, then

$$
\begin{aligned}
& i \gamma_{1} @\left(n\left(s \alpha_{2}\right) \bigoplus\left(i \alpha_{1} \bigotimes \beta_{1}(y)\right)\right) \\
& \quad \leq C(A, B ; S)(y) \\
& \quad \leq s \gamma_{2} @\left(n\left(i \alpha_{1}\right) \bigoplus\left(s \alpha_{2} \bigotimes \beta_{2}(y)\right)\right)
\end{aligned}
$$

where $\otimes, \bigoplus$ and $n$ are, respectively, a $t$-norm, a $t$-conorm and a fuzzy complement.

3) If $\rightarrow$ is a $t$-norm $\otimes$ implication, then

$$
\begin{aligned}
i \gamma_{1} @\left(i \alpha_{1} \bigotimes \beta_{1}(y)\right) & \leq C(A, B ; S)(y) \\
& \leq s \gamma_{2} \subseteq\left(s \alpha_{2} \bigotimes \beta_{2}(y)\right) .
\end{aligned}
$$

Proof:

1) Using the properties of $S$-implications, $R$-implications, and $t$-norms, or $t$-conorms, we have

$$
\begin{aligned}
& i \gamma_{1} @\left(s \alpha_{2} \rightarrow \beta_{1}(y)\right) \\
& =\sup _{x \in U}\left(\inf _{x \in U}\left(\gamma_{1}(x)\right) \subset\left(\sup _{x \in U}\left(\alpha_{2}(x)\right) \rightarrow \beta_{1}(y)\right)\right) \\
& \leq \sup _{x \in U}(S(x) \subseteq(A(x) \rightarrow B(y))) \\
& =C(A, B ; S)(y) \\
& \leq \sup _{x \in U}\left(\sup _{x \in U}\left(\gamma_{2}(x)\right) \subset\left(\inf _{x \in U}\left(\alpha_{1}(x)\right) \rightarrow \beta_{2}(y)\right)\right) \\
& =s \gamma_{2} \bigodot\left(i \alpha_{1} \rightarrow \beta_{2}(y)\right) \text {. }
\end{aligned}
$$

The inequalities of 2) and 3) are verified directly from the monotonicity of the $t$-norm, $t$-conorm, and the fuzzy complement $n$. We omit the details.

When the $t$-norm, $t$-conorm, and the fuzzy complement $n$ are continuous in Theorem 4 , the fuzzy reasoning scheme using the Zadeh's CRI method with (c) (some $t$-norm or $t$-conorm) and an $S$-implication or an $Q L$-implication, or a $t$-norm implication is stable. When the oscillation limits of an input $(A, B, S)$ in some fuzzy reasoning scheme using Zadeh's CRI methods are gradually contracted, i.e., there exists a fuzzy interval perturbation sequence $\left(\left[\alpha_{1 n}, \alpha_{2 n}\right],\left[\beta_{1 n}, \beta_{2 n}\right],\left[\gamma_{1 n}, \gamma_{2 n}\right]\right)$ of the input $(A, B, S)$ such that

$$
\begin{aligned}
& \lim _{n \rightarrow \infty} \sup _{x \in U}\left(\alpha_{2 n}(x)-\alpha_{1 n}(x)\right)=0 \\
& \lim _{n \rightarrow \infty} \sup _{y \in V}\left(\beta_{2 n}(y)-\beta_{1 n}(y)\right)=0 \\
& \lim _{n \rightarrow \infty} \sup _{x \in U}\left(\gamma_{2 n}(x)-\gamma_{1 n}(x)\right)=0
\end{aligned}
$$

hold, then the output values converge stably to some value.

On the other hand, the fuzzy reasoning schemes using Zadeh's CRI methods with (c) ( $t$-norm or $t$-conorm) and some $R$-implications are not stable.

\section{CONCLUSION}

We know that a fuzzy reasoning scheme applied to practice is probably perturbed by "noises" in various ways. In addition to many proximity measures of fuzzy sets, the simple perturbation and interval perturbation may effectively simulate such "noises" as well. In fact, Ying's maximum $\varepsilon$ perturbation of a fuzzy set [14] is a simple perturbation, some error estimation of the output values in fuzzy reasoning is more precise using the approach of this correspondence. Pappis' proximity measure of two fuzzy sets [12], Hong and Hwang's $\alpha$-similarity of two fuzzy sets [13], Cai's $\delta$-equality of two fuzzy sets [15] and Ying's maximum $\varepsilon$ perturbation of a fuzzy set are all formulated by the interval perturbation of the fuzzy sets. Therefore, in certain sense this correspondence is a further development of the previous work. On the other hand, we take into account the effects of realistic noise and accurately evaluate the output errors of fuzzy reasoning. Therefore, we may choose a fuzzy reasoning scheme according to the requirement of the output errors in applications.

\section{ACKNOWLEDGMENT}

The authors would like to thank the referees for the invaluable comments and suggestions. The proof of 1) in Theorem 4 is due to the anonymous referee.

\section{REFERENCES}

[1] L. A. Zadeh, "The concept of a linguistic variable and its applications to approximate reasoning, I,II,III," Inform. Sci., vol. 8, pp. 199-249, 1974

[2] D. Dubois and H. Prade, "Fuzzy sets in approximate reasoning, part 1: Inference with possibility distributions," Fuzzy Sets Syst., vol. 40, pp. 143-202, 1991.

[3] H. Nakanishi, I. B. Turksen, and M. Sugeno, "A review and comparison of six reasoning methods," Fuzzy Sets Syst., vol. 57, pp. 257-294, 1993.

[4] E. S. Lee and Q. Zhu, Fuzzy and Evidence Reasoning. Hiedelberg, Germany: Physica-Verlag, 1995.

[5] J. W. Guan and D. A. Bell, "Approximate reasoning and evidence theory," Inform. Sci., vol. 96, pp. 207-235, 1997.

[6] W. H. Hsiao, S. M. Chen, and C. H. Lee, "A new interpolative reasoning methods in sparse rule-based systems," Fuzzy Sets Syst., vol. 93, pp. 17-22, 1998.

[7] J. L. Castro, E. Trillas, and J. M. Zurita, "Non-monotic fuzzy reasoning," Fuzzy Sets Syst., vol. 94, pp. 217-225, 1998.

[8] Y. Liu and E. E. Kerre, "An overview of fuzzy quantifiers(II): Reasoning and applications," Fuzzy Sets Syst., vol. 95, pp. 135-146, 1998.

[9] M. S. Ying, “A logic for approximate reasoning," J. Symb. Logic, vol. 59, pp. 830-837, 1994. 
[10] G. J. Wang, “On the logic foundation of fuzzy reasoning," Inform. Sci., vol. 117, pp. 47-88, 1999

[11] L. X. Wang, A Course in Fuzzy Systems and Control. Englewood Cliffs, NJ: Prentice-Hall, 1997.

[12] C. P. Pappis, "Value approximation of fuzzy systems variables," Fuzzy Sets Syst., vol. 39, pp. 111-115, 1991.

[13] D. H. Hong and S. Y. Hwang, "A note on the value similarity of fuzzy systems variables," Fuzzy Sets Syst., vol. 66, pp. 383-386, 1994.

[14] M. S. Ying, "Perturbation of fuzzy reasoning," IEEE Trans. Fuzzy Syst., vol. 7, no. 5, pp. 625-629, Oct. 1999.

[15] K. Y. Cai, "Robustness of fuzzy reasoning and $\delta$-equalities of fuzzy sets," IEEE Trans. Fuzzy Syst., vol. 9, no. 5, pp. 738-750, Oct. 2001.

[16] M. S. Ying, "Implication operators in fuzzy logic," IEEE Trans. Fuzzy Syst., vol. 10, no. 1, pp. 88-91, Feb. 2002.

[17] S. Weber, "A general concept of fuzzy connectives,Negations and implication based on t-norms and t-conorm," Fuzzy Sets Syst., vol. 11, pp. 115-134, 1983.

[18] D. Boixader and L. Godo, , E. H. Ruspini, P. P. Bonissone, and W. Pedrycz, Eds., "Fuzzy inference," in Handbook of Fuzzy Computation. Philadelphia, PA: Inst. Phys., 1998.

[19] R. Fullér, , C. Carlsson, Ed., "On fuzzy reasoning schemes," in The State of the Art of Information Systems Applications in 2007. Turku, Finland: TUCS General Publications, 1999, vol. 16, pp. 85-112.

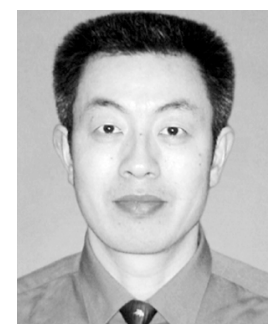

Guosheng Cheng received the B.S. degree from Huaibei Coal Industry Teacher's College, Huaibei, China, in 1986, and the M.S. and Ph.D. degrees from Xi' an Jiaotong University, Xi' an, China, in 1996 and 2000, respectively.

$\mathrm{He}$ is a Professor in the Department of Mathematics, Nanjing University of Information Science and Technology, Nanjing, China. His research interests include intelligent computation and computability of the measures.

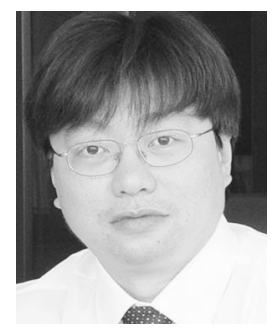

Yuxi Fu received the B.S. degree from Tongji University, Shangai, China, and the Ph.D. degree from the University of Manchester, Manchester, U.K., in 1986 and 1992, respectively.

$\mathrm{He}$ is a Full Professor with the Department of Computer Science, Shanghai Jiaotong University, Shangai, China. His research interest lies in theoretical computer science, especially in type theory and concurrency theory. His recent work has focused on the studies of process calculi, such as pi-calculus and chi-calculus. 